University of Wollongong

Research Online

Faculty of Informatics - Papers (Archive)

Faculty of Engineering and Information

Sciences

7-5-2006

\title{
Performance Comparison of UWB Hopping Codes in a Multi-User Rich Scattering Environment
}

\author{
K. Popovski \\ University of Wollongong, keni@uow.edu.au \\ Beata J. Wysocki \\ University of Wollongong, bjw@uow.edu.au \\ Tadeusz A. Wysocki \\ University of Wollongong, wysocki@uow.edu.au
}

Follow this and additional works at: https://ro.uow.edu.au/infopapers

Part of the Physical Sciences and Mathematics Commons

\section{Recommended Citation}

Popovski, K.; Wysocki, Beata J.; and Wysocki, Tadeusz A.: Performance Comparison of UWB Hopping Codes in a Multi-User Rich Scattering Environment 2006.

https://ro.uow.edu.au/infopapers/418

Research Online is the open access institutional repository for the University of Wollongong. For further information contact the UOW Library: research-pubs@uow.edu.au 


\title{
Performance Comparison of UWB Hopping Codes in a Multi-User Rich Scattering Environment
}

\author{
Abstract \\ Ultra Wideband is a short-range wireless communication technique which has seen increased interest \\ over the past decade. Having several methods of implementation, a key issue will always be the \\ avoidance of multi-user interference. For time-hopped Ultra Wideband, this is achieved through the use of \\ different codes assigned to each user, performing time-hopped code division multiple access. This paper \\ presents a performance comparison of several time hopping sequence construction methods. It presents \\ correlation results between sequences, and simulation results in a Gaussian noise degraded, scattering \\ rich multi-user environment. Simulations are based on the IEEE channel model for UWB communications.

\section{Disciplines} \\ Physical Sciences and Mathematics

\section{Publication Details} \\ This paper was originally published as: Popovski, K, Wysocki, BJ Wysocki, TA, Performance Comparison \\ of UWB Hopping Codes in a Multi-User Rich Scattering Environment, IEEE 63rd Vehicular Technology \\ Conference 2006 (VTC 2006-Spring), Melbourne, Australia, 7-10 May 2006, 4, 1864-1868. Copyright 2006 \\ IEEE.
}




\title{
Performance Comparison of UWB Hopping Codes in a Multi-User Rich Scattering Environment
}

\author{
K. Poporski, B. J. Wysocki, T. A. Wysocki (Senior Member IEEE) \\ School of Electrical, Computer and Telecommunications Engineering \\ University of Wollongong \\ Northfields Ave, Wollongong, NSW, 2522, Australia \\ keni@uow.edu.au, beata@elec.uow.edu.au, wysocki@uow.edu.au
}

\begin{abstract}
Ultra Wideband is a short-range wireless communication technique which has seen increased interest over the past decade. Having several methods of implementation, a key issue will always be the avoidance of multi-user interference. For time-hopped Ultra Wideband, this is achieved through the use of different codes assigned to each user, performing time-hopped code division multiple access.

This paper presents a performance comparison of several time hopping sequence construction methods. It presents correlation results between sequences, and simulation results in a Gaussian noise degraded, scattering rich multi-user environment. Simulations are based on the IEEE channel model for UWB communications.
\end{abstract}

Keywords: Ultra Wideband, time hopping, Hamming correlation, multi-user interference

\section{INTRODUCTION}

Ultra Wideband (UWB) is an emerging wireless technology, characterised by having a fractional bandwidth of more than $20 \%$, or a bandwidth greater than $500 \mathrm{MHz}$ [1]. The two competing standards for UWB are: orthogonal frequency division multiplexing (UWB-OFDM) and direct sequence (DS -UWB). UWB-OFDM is based on traditional OFDM communications, utilising the frequency spectrum such that it appears an entire $528 \mathrm{MHz}$ band is being simultaneously used. DS-UWB is pulse based, sending out encoded waveforms which occupy a large bandwidth over several gigahertz [2]

This paper covers multiple access issues within time hopped UWB (TH-UWB), similar in implementation to DS-UWB. Pulses are either delayed in time (pulse position modulation (PPM)) or changed in amplitude (pulse amplitude modulation (PAM)) for the encoding of data. Users are multiplexed through a delay of the pulses (once again if position modulation is used). This paper deals with PPM TH-UWB. Sequence designs are focused on time hopping codes; while application to frequency hopping is viable, their correlation properties will be altered [3].

Shown in (1) [4] is the signal transmitted for a single user in a time hopped system, where the data is mapped using binary PPM. A shift of the pulse is used to encode data, indicated by the $\delta$ shift in the equation.

$$
s^{(i)}(t)=\sum_{m=-\infty}^{\infty} w\left(t-m T_{f}-c_{m}^{(i)} T_{c}-\delta b_{m}^{(i)}\right)
$$

Each bit is transmitted in a single frame, which is of length $T_{f}$. This frame is then segmented into equally spaced intervals called 'chips' of duration $T_{c}$. The hopping sequence is then used to determine which chip within each frame a particular user will be occupying. If two users occupy the same chip, a collision or 'hit' has occurred.

Proper design of orthogonal codes decreases the possibility of multi-user interference, and lowers the effects of multipath interference on communications [3]. Sequences which have low enough cross-correlation values also allow for the multiuser interference within a system to be modelled as a random Gaussian process [5].

There are various aspects which must be considered in the design of orthogonal sequences. These include:

- efficient and even utilisation of the available channels;

- not occupying the same channel for many repetitions;

- avoidance of sub-sequences between user patterns;

- sequences not being too short, in order to increase the number of patterns possible, although not too long due to the possibility of causing synchronisation issues.

The four main parameters in sequence characterisation are: the size of the alphabet, also referred to as the cardinality of the code; the period of the sequences; the size of the set of sequences possible; and the correlation values between sequences. The maximum number of users possible within a TH-UWB system is equal to the cardinality of the code, this indicating the number of chips that exist within a frame.

This paper presents a performance comparison of various time hopping codes. Simulations were conducted using a modified UWB system, initially implemented by Di Benedetto and Giancola [6]. The time hopped, pulse position modulated simulation accounts for Gaussian distributed channel noise, multi-user interference, and channel modelling through the IEEE UWB channel model. This model is based upon the Saleh and Valenzuela (SV) system of 1987, where multipath contributions from each pulse arrive at the receiver in clusters. The original SV model was modified by the IEEE, adopting log-normal distributions for multipath amplitudes, and lognormal fluctuations of the total multipath gain [7].

Three types of receivers were tested: All-Rake (ARake), which collects all multipath components; Selective-Rake (SRake), which collects the $L$ multipath components which 
have the largest amplitudes; and Partial-Rake (PRake), which collects the first $L$ components received [8].

The rest of this paper is organised as follows: Section II provides an overview of the comparison measures that were used between sequences; Section III outlines the sequence design methods tested; Section IV covers simulation parameters and results obtained; and finally Section V presents all concluding statements.

\section{Performance Comparison Measures}

\section{A. Periodic Hamming Correlation}

Recalling that a sequence is a series of numbers signifying which chip a user's pulse will occur in for a certain time frame, a 'hit' for this correlation occurs when two or more users attempt to occupy the same time segment. The Hamming correlation is a measure of the number of hits between sequences for a certain relative time delay. It is defined by (2) [9], which has been modified to account for sequences of different lengths.

$$
\begin{aligned}
& H_{X Y}(\tau)=\frac{1}{N_{p}} \sum_{i=0}^{N_{p}-1} h\left(x_{i}, y_{i+\tau}\right) \quad 0 \leq \tau \leq N_{p}-1 \\
& \text { where: } h(a, b)= \begin{cases}0, & a \neq b \\
1, & a=b\end{cases}
\end{aligned}
$$

$\tau$ represents the time asynchronicity between the patterns, and $N_{p}$ represents the periodicity of the sequences. Since patterns within different families may be of different length, division by $N_{p}$ is used to normalise the correlation results. Results were obtained using cyclically shifted sequences, averaged over all possible sequence pairs within a family.

It should be noted that the 'difference equation', used as a correlation measure for frequency hopped codes [10], reduces to the Hamming cross-correlation for a constant Doppler shift.

\section{B. Direct Sequence Correlation}

Utilised as a correlation measure for sequences which were initially designed for use in Direct Sequence systems, defined by (3) [11]. Once orthogonal codes were determined, they were mapped into integer sequences which may be applied to UWB time hopping. The Hamming correlation measure was then strictly used. $N$ represents the length of the codes, with $\tau$ being their asynchronicity.

$$
R(\tau)=\sum_{i=0}^{N-1} x_{i} y_{i+\tau}^{*}
$$

\section{Sequence Construction Techniques}

\section{A. Introduction}

Time hopping sequences may be designed through varied procedures. These include dependence on a predefined distribution for the resulting values, an equation relating various parameters of the sequences (known as a placement operator), or a direct mapping from the complex domain to the integer domain. The 'user number' is a crucial part of the design procedure. Assuming uniqueness over all users, it is required to obtain a sequence from a family of patterns generated from each design.

Given next are the design procedures for ten families of time hopping codes. Common parameters are: $a$ the user number, $k$ the iteration number for a code, and $p$ chosen as a prime value.

\section{B. Random Sequences}

Generation of random numbers with a uniform distribution over 0 to 1 , which are then scaled in order to attain a series of randomly generated hopping sequences. These are used as a control set for the simulation, based upon the assumption that sequences which are designed with some underlying structure to achieve orthogonality must on average have an improved performance than a set of sequences which have no structure.

\section{Linear Congruence Codes}

Linear sequences are designed based entirely on two parameters: user number and iteration number. They are defined through the following placement operator [12]:

$$
y(k)=a k(\bmod p)
$$

For simulation purposes, the cardinality and the periodicity of the codes are chosen to be equivalent, equal to the value of $p$. This method produces a family of $p$ different sequences.

\section{Cubic Congrience Codes}

Based upon the user number and the cubic of the iteration number through the placement operator [13]:

$$
y(k)=a k^{3}(\bmod p)
$$

Provides a set of $p-l$ codes, with a periodicity of $p$ and a cardinality of $p-1$. However, a restriction that exists on these cubic congruence codes is that the value of $p$, which is restricted to being a prime value, is also restricted to satisfy (6), with $m$ being an integer. This is in order to generate a full set of codes, using all of the available time slots equally. For this simulation, $m$ was set equal to three, in order to have the value of $p$ equal to 11 (see Section IV).

$$
p=3 m+2
$$

\section{E. Quadratic Congruence Codes - Construction 1}

These codes are constructed through the placement operator in (7) [14]. The parameters $\alpha$ and $\beta$ may be varied in order to obtain a larger set of sequences. However, for purposes of this paper's simulation, the value of $\beta$ was set to equal the iteration number $k$. Also, the value of $\alpha$ was initially set to 1 , then incremented when the user number exceeded $p-1$.

$$
y(k)=\left[a(k+\alpha)^{2}+\beta\right](\bmod p)
$$

The periodicity of the codes is set to equal $p$ and cardinality equal to $p-1$, the placement operator may be used to obtain a set of $(p-1) p$ sequences.

\section{F. Quadratic Congruence Codes - Construction 2}

Similar to the quadratic congruence codes of 'Construction 1 '. These are defined through the placement operator of (8), where $p$ is chosen as an odd prime value restricted to being greater than or equal to three [15].

$$
y(k)=a k(k+I) / 2(\bmod p)
$$


One disadvantage of these codes is they do not use all available time slots evenly. Consequently, the system's performance degrades as some chips are under-utilised.

\section{G. Hyperbolic Congruence Codes}

These sequences are based upon a hyperbolic congruence between the user number and the iteration number through the placement operator [10]:

$$
y(k)=a / k(\bmod p)
$$

This family of codes allows a maximum of $p-1$ users, with a cardinality of $p-l$ and a periodicity of $p$. However, the computation to determine the value of each member of a sequence may not be conducted by direct division by the parameter $k$. Rather, the inverse of $k$ must first be determined, found by considering the Galois field that $k$ belongs to. Here, any operation that is conducted on a member of the field results in a value within the field itself.

\section{H. Additional Sequences}

These simple codes are constructed through (10), where the user number is added to the iteration number, and contained by the prime $p$ [3]. The cardinality of the codes is $p-1$, the periodicity is $p$, and there are $p$ sequences within this family.

$$
y(k)=[k-a-1](\bmod p)
$$

It should be noted that all sequences produced are cyclically shifted versions of the codes in the same set.

\section{Chu Sequences}

Developed for use in Direct Sequence spreading, where the patterns are designed using the complex primitive $N$ th roots of unity, with $N$ being the length of the sequences. Sequence elements are given by [11]:

$$
y(k)=\left\{\begin{array}{ll}
e^{\frac{i \pi}{N} a(k+1) k} & N \text { odd }
\end{array} \quad 0 \leq k<N\right.
$$

The numbers that are relative prime to and less than $N$ were determined, and used as the user numbers. The number of users possible is limited by the amount of numbers relatively prime to $N$, equivalent to the 'Euler Totient' function. Once the Direct Sequence code was determined, a direct mapping from the complex to the integer domain was conducted.

The number of possible sequences and their cardinality are equivalent and equal to $N-I$. Unfortunately, the sequences that are designed are palindromes, and as such increase the possibility of sequence prediction.

\section{J. One-Coincidence Sequences}

Designed through a simple relationship between the user number, the iteration number, and a primitive element $r$, given by the equation [11]:

$$
y(k)=r^{k}+a(\bmod p)
$$

This method requires a Galois field of size $p$, and the finding of a primitive element ( $r$ ) of the field (which when raised to the powers from 0 to $p-2$ provides all elements of the field). For this simulation, $r$ was chosen as 2 , this being a primitive element of a Galois field of size 11 (see Section IV).
The maximum number of users is $p$, and the periodicity of the codes is $p-1$. The average Hamming cross-correlation function is defined by $\left(N_{p}-1\right) / N_{p}$, with $N_{p}$ being equal to the periodicity of the codes.

\section{K. Reed-Solomon Sequences}

These sequences are designed through a subset of ReedSolomon codewords which are commonly applied in error correction coding [16-17], characterised by allowing a controlled amount of correlation between sequences.

The design of these codes requires the determination of two parameters: $Q$ - the number of time slots available; and $s$ the maximum number of permitted in-phase hits between sequences. However, the out-of-phase correlation between sequences may result in as many as $Q-1$ hits.

There are $\dot{Q}^{s-1}$ codewords possible, each of length $Q-1$. The codewords $\left(f_{x}\right)$ are obtained through the multiplication of the $(s+1) \times 1$ element matrix $\mathbf{n}$ by the generator matrix $G$, as in (13). $G$ is defined as the primitive element of $Q$ raised to the power of $(i-1) j$, with $i$ and $j$ representing the row and column of $G$ respectively. Elements of $\mathbf{n}$ may range from $\theta$ to $Q-1$.

$$
f_{x}=\left[n_{0}, n_{1}, \ldots, n_{s}\right] G
$$

Due to the fact that the cyclically time shifted versions of a codeword are sequences themselves, the complete set of ReedSolomon codewords is a poor choice of hopping patterns. Thus a subset with better properties is found by limiting the values $n_{0}$ and $n_{1}$ [17]. Firstly, $n_{0}$ is chosen constant for all sequences within a set, since it increases all sequence values by the same amount in accordance with the constant top row of $G$; and secondly a nonzero value is chosen for $n_{1}$, since the second row of $G$ consists of the consecutive numbers from 1 to $\mathrm{Q}$, and will attain a certain level of uniqueness between sequences. All other possible combinations are assigned to the remaining elements of $\mathbf{n}$, forming the sequences within the set. $Q(Q-I)$ sets may be obtained by choosing different values for $n_{0}$ and $n_{1}$.

For simulation purposes, $Q$ was set to 11 , its primitive element chosen as 2 , and $s$ set to 2 . This allows 11 different sequences to be generated for each set. $n_{0}$ was set to 0 and $n_{1}$ to 1 in accordance with the aforementioned restrictions.

\section{Simulation}

\section{A. System Parameters}

The parameter $p$ was chosen to be 11 for all time hopping code designs. This limits the number of chips in a frame to 11 , and the maximum number of users to 10 for some sequences. For comparable results, all sequences were tested for two users (one transmitting and one interfering) and 10 users (one transmitting and nine interfering).

The multipath channel parameters were set to simulate a line-of-sight transmission, within a 0 to 4 meter range, for all users. This affects the amplitude of the dispersed paths, and the multipath spread. For the Selective-Rake and Partial-Rake receivers, 5 multipath components were used $(\mathrm{L}=5)$.

The chip time $T_{c}$ was set to $30 \mathrm{~ns}$, and the frame time $T_{f}$ to $330 \mathrm{~ns}$. All tests performed had each pulse represent a single bit of information. In accordance with IEEE 802.15 UWB 
standards [18], packet sizes of 1024 octets were used, with a total of 1007616 bits being sent. Channel parameters and user asynchronicity were randomly generated after each full packet was transmitted.

Chip synchronicity was assumed throughout the simulation, proven to provide a worst case performance bound [19].

\section{B. Results}

Hamming correlations conducted on all time hopping sequences within a family returned the maximum, minimum, and average number of in-phase and out-of-phase hits. Average values for all sequences were generally 0.1 . While some sequences may have exhibited smaller minimum values, this was offset by larger maximum correlation results between other sequences within the family. 'Reed-Solomon' codes were the exception to this, having an average and maximum out-ofphase correlation of 0.1 , and a minimum of usually zero.

Shown in Fig. 1(a) are the bit error rate (BER) versus signal to noise ratio (SNR) plots for each of the Rake receiver types for the two user case, and Fig. 1(b) shows the 10 user case. Initial inspection of the results reveals all depict the transition from noise dominance to interference dominance as the SNR is increased. At low SNR values, decoding errors are mainly caused by noise interfering with the transmission; while at high SNR values errors result from the interference between users.

There exists a minimum BER in each system, caused by multi-user interference, shown as a plateau in the high SNR regions. As expected, the BER plateau is lower for the two user case (approximately $10^{-3}$ ) than in the 10 user case (approximately $10^{-2}$ ), due to the decreased level of interference. A significant difference can also be seen between receiver types. The All-Rake receiver provides optimal performance (lowest BER levels), since it combines all scattered components in the calculation of encoded data. The SelectiveRake receiver provides a relatively worse performance, and the Partial-Rake gives the worst results.

An abnormality in the results exists within the two user Selective-Rake case, for the randomly generated codes. Their BER curve falls well below the other hopping codes. This leads to the conclusion that in a system which is exhibiting low levels of utilisation, it is possible to use unstructured codes for users. However, in a fully utilised system (Fig. 1(b)) it can be seen that random codes perform relatively poorly, and a structured user coding is required to avoid simultaneous transmissions.

It has been determined that in an under utilised system $(20 \%$ of its capacity), both constructions for 'Quadratic' codes, together with 'Reed-Solomon' codes perform well, although so do unstructured random codes. In the 10 user case (full utilisation), 'Reed-Solomon' codes provide paramount performance, relative to other constructions.

It should be noted that although 'Additional' codes also performed relatively well, results were unpredictable. Their adoption into wireless devices is however not viable due to all codes being cyclical shifts of one another.

\section{CONCLUSION}

A system implementing time-hopped UWB has been used in a performance comparison for ten sequence constructions, in two user and 10 user scenarios. It was determined that for a system exhibiting low levels of utilisation (two user case) structured codes have the same performance as randomly generated codes. However, this is not true for the fully utilised system (10 user case), where structured codes out-performed random sequences. It was found that 'Reed-Solomon' codes displayed optimal results, as anticipated by correlation results.

Future work into this area may extend the range of sequence constructions tested, and also investigate relative performance when more than one pulse is transmitted per data bit.

\section{REFERENCES}

[1] - FCC Document 00-163: "Revision of Part 15 of the Commission's Rules Regarding Ultra-Wideband Transmission Systems", April 22, 2002, ET Docket No. $98-153$

[2] - Sumner Lemon, "Standards deadlock hits UWB - The market will have to decide", IDG News Service, 17 May 2005 ,

http://www.techworld.com/applications/news/index.cfm?NewsID $=3674$

[3] - I. Guvenc, H. Arslan, "Design and performance analysis of TH sequences for UWB-IR systems", Wireless Communications and Networking Conference, 2004. WCNC, 2004 IEEE, Volume: 2, 21-25 March 2004, pp: 914 - 919 Vol.2 [4] - T. Erseghe, "Time-Hopping Patterns derived from Permutation Sequences for Ultra-Wide-Band Impulse-Radio Applications", University di Padova, accessed on $25 / 3 / 05$, accessible at http://www.dei.unipd.it/ erseghe/docs/uwbwseas02.pdf

[5] - R. Z. Scholtz, "Multiple access with time-hopping impulse modulation", Military Communications Conference, 1993, MILCOM'93, Conference record: 'Communications on the Move', IEEE, Vol. 2, 11-14 Oct. 1993, pp. $447-450$ [6] - M-. G. Di Benedetto, G. Giancola, "Understanding Ultra Wide Band Radio Fundamentals", Prentice Hall Professional Technical Reference, 2004

[7] - Saleh, A.; Valenzuela, R., "A Statistical Model for Indoor Multipath Propagation", IEEE Journal on Selected Areas in Communications, Vol. 5, Iss. 2, Feb 1987, pp: $128-137$

[8] - M. Z. Win, Z. A. Kostic, "Virtual path analysis of selective Rake receiver in dense multipath channels", IEEE Commun. Letter, Vol. 3, Nov. 1999, pp: 308-310

[9] - M. S. Iacobucci, M. -G. Di Benedetto, "Multiple access design for impulse radio communication systems", IEEE International Conference on Communications, 2002. ICC 2002, Vol. 2, 28 April, pp: $817-820$

[10] - O. Moreno, S. V. Maric, "A new family of frequency-hop codes", IEEE Transactions on Communications, Vol. 48, Iss. 8, Aug. 2000, pp: 1241 - 1244 [11] - P. Fan, M. Darnell, "Sequence Design for Communications Applications", Research Studies Press Ltd., Somerset, England, 1996

[12] - A. V. Jovancevic, E. L. Titlebaum, "New coding schemes for increased number of users or messages in frequency-hopped multilevel FSK", Vehicular Technology Conference, 1996. Mobile Technology for the Human Race',, IEEE $46^{\text {th }}$, Vol. 3, 28 April 1996, pp: $1732-1735$

[13] - S. V. Maric, E. L. Titlebaum, "Frequency hop multiple access codes based upon the theory of cubic congruences", IEEE Transactions on Aerospace and Electronic Systems, Vol. 26, Iss. 6, Nov. 1990,pp: 1035-1039

[14] - Z. Kostic, E. L. Titlebaum, S. V. Maric, "The design of new optical codes and time-hopping patterns for synchronous spread-spectrum codedivision multiple-access communication systems", IEEE International Conference on Communications, 1991. ICC 91, Conference Record, 23-26 June 1991, Vol. 2, pp: 585 - 589

[15] - E. L. Titlebaum, S. V. Maric, J. R. Bellegarda, "Ambiguity properties of quadratic congruential coding", IEEE Transactions on Aerospace and Electronic Systems, Vol 27, Iss 1, Jan. 1991, pp: 18 - 29

[16] - I. S. Reed, G. Solom on, "Polynomial Codes over Certain Finite Fields", $J$ Soc. Indust. Appl. Math., Vol. 8, No. 2, June 1960

[17] - R. M. Mersereau, T. S. Seay, "Multiple Access Frequency Hopping

Patterns with Low Ambiguity", IEEE Transactions on Aerospace and

Electronic Systems, Vol. 17, No. 4, July 1981, pp. 571-578 
[18] - M. Welborn, "Proposal Comparison Summary", IEEE P802 15 Working Group for Wireless Personal Area Networks (WPANs), May 12, 2004
[19] - J. A. Salehi, "Code division multiple-access techniques in optical fiber networks. I. Fundamental principles", IEEE Transactions on Communications, Vol. 37, Iss. 8, Aug. 1989, pp: 824 - 833

(b)
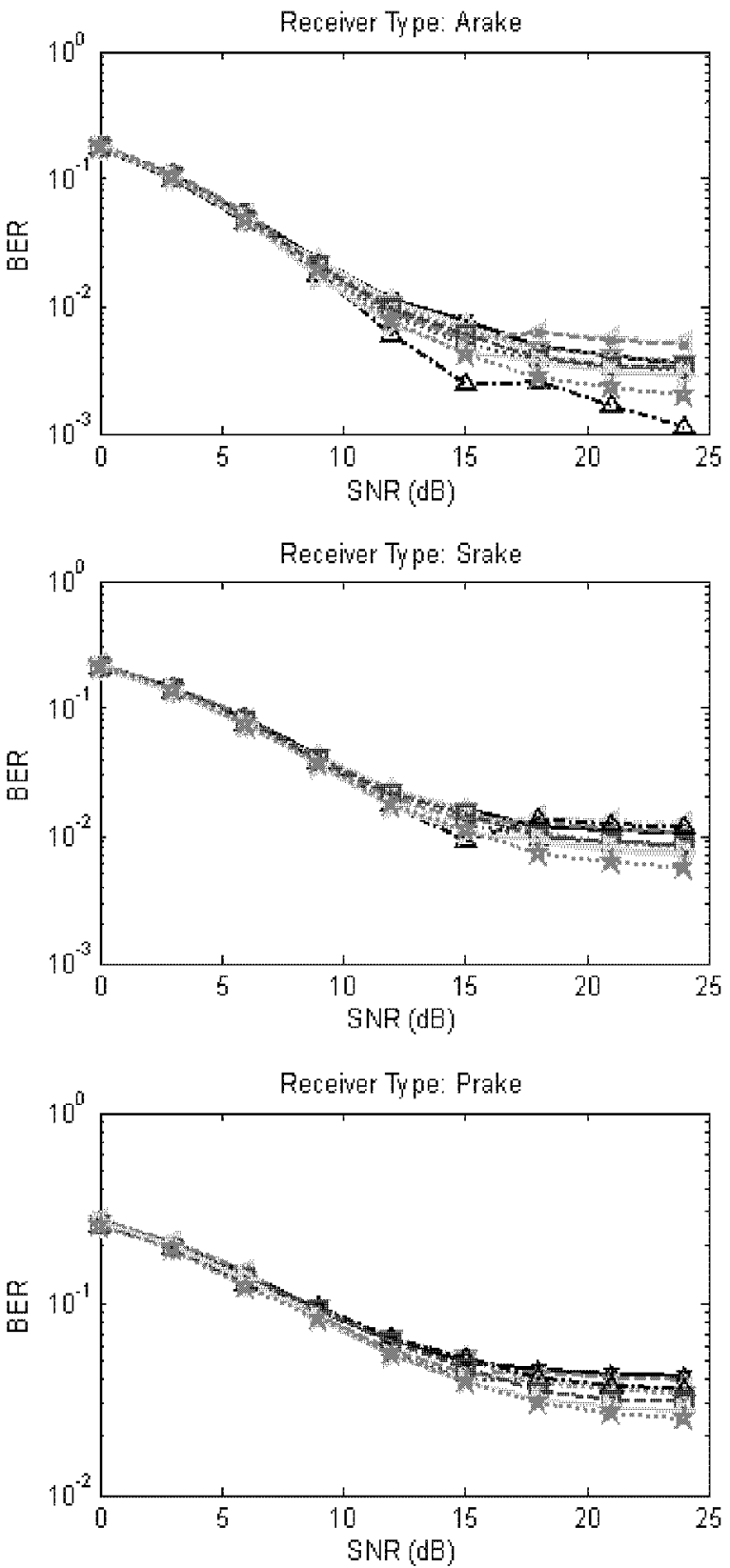

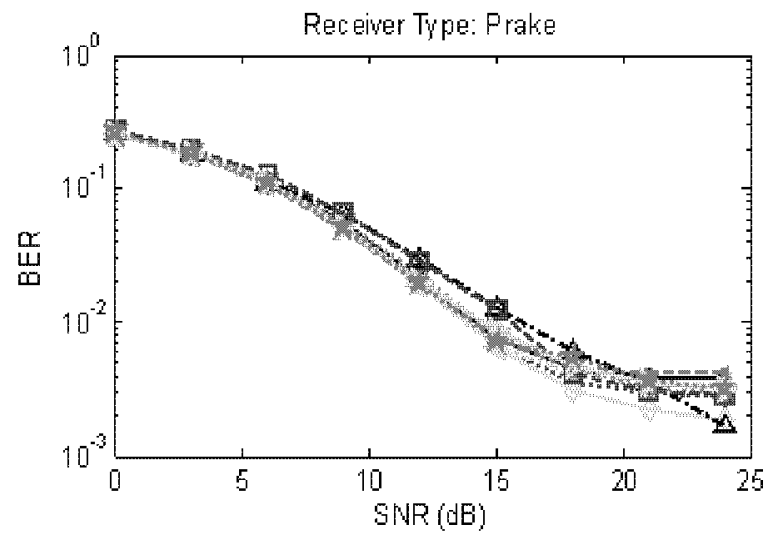

Figure 1: BER vs SNR plots for time-hopped UWB with ten time hopping sequence construction techniques (a): 2 user case, (b): 10 user case

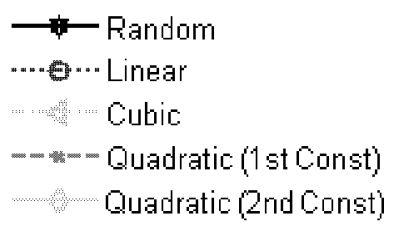

$$
\begin{aligned}
& \text { axis } \\
& \text { - - A - Aditional }
\end{aligned}
$$

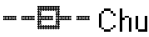

$$
\begin{aligned}
& \text { One-Coincidence }
\end{aligned}
$$

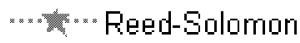

